PROCEEDINGS OF THE

AMERICAN MATHEMATICAL SOCIETY

Volume 137, Number 1, January 2009, Pages 171-178

S 0002-9939(08)09448-3

Article electronically published on July 1, 2008

\title{
MULTIPLICATIVE BIJECTIONS OF SEMIGROUPS OF INTERVAL-VALUED CONTINUOUS FUNCTIONS
}

\author{
JESÚS ARAUJO \\ (Communicated by N. Tomczak-Jaegermann)
}

\begin{abstract}
We characterize all compact and Hausdorff spaces $X$ which satisfy the condition that for every multiplicative bijection $\varphi$ on $C(X, I)$, there exist a homeomorphism $\mu: X \longrightarrow X$ and a continuous map $p: X \longrightarrow(0,+\infty)$ such that

$$
\varphi(f)(x)=f(\mu(x))^{p(x)}
$$

for every $f \in C(X, I)$ and $x \in X$. This allows us to disprove a conjecture of Marovt (Proc. Amer. Math. Soc. 134 (2006), 1065-1075). Some related results on other semigroups of functions are also given.
\end{abstract}

\section{INTRODUCTION}

For a compact and Hausdorff space $X$, let $C(X, I)$ denote the semigroup of all continuous functions on $X$ taking values in the closed unit interval $I=[0,1]$. Motivated by a result of L. Molnár (see [6]), J. Marovt studied the form of multiplicative bijections on $C(X, I)$ in a special case, namely, when $X$ satisfies the first axiom of countability (see [4). He proved that if $\varphi$ is such a map, then there exist a homeomorphism $\mu: X \longrightarrow X$ and a continuous map $p: X \longrightarrow(0,+\infty)$ such that

$$
\varphi(f)(x)=f(\mu(x))^{p(x)}
$$

for every $f \in C(X, I)$ and $x \in X$. We call maps of this form standard. Marovt conjectured that every multiplicative bijection on $C(X, I)$ was necessarily standard and that the assumption on $X$ of being first countable could be dropped.

In this paper we prove that Marovt's conjecture does not hold. In fact we give a characterization of spaces $X$ for which there exists a multiplicative bijection of $C(X, I)$ that is not standard. Roughly speaking, we prove that this is the case if and only if $X$ is the Stone-Čech compactification of a proper subset (see Theorem 2.2).

We study a more general case, that of multiplicative bijections between semigroups of functions. Of course, a multiplicative bijection between arbitrary semigroups of $C(X, I)$ need not be of the above form, even if they separate points. A simple example is the following: take $X$ having only one point, so each semigroup of $C(X, I)$ can be identified with a semigroup of $I$. Consider for instance $s=1 / 2$, $t=1 / 3$, and $\mathcal{A}:=\left\{s^{n} t^{m}: n, m \in \mathbb{N}\right\}$. It is now clear that the map sending each $s^{n} t^{m}$ into $s^{m} t^{n}$ is multiplicative and bijective, but cannot be described as above.

Received by the editors October 26, 2007, and, in revised form, December 10, 2007.

2000 Mathematics Subject Classification. Primary 46J10; Secondary 46E05, 54D35.

This research was partially supported by the Spanish Ministry of Science and Education (Grant number MTM2006-14786).

(C)2008 American Mathematical Society 171

Reverts to public domain 28 years from publication 
Nevertheless, our technique can be used for semigroups other than $C(X, I)$. In fact, it works if we just require these semigroups $\mathcal{A} \subset C(X, I)$ to satisfy the following three properties (where, as usual, if $f \in C(X, I), \operatorname{coz} f$ denotes the set $\{x \in X: f(x) \neq 0\}$, and $\operatorname{supp} f$ denotes its closure):

Property 1: Given any $x \in X$ and any neighborhood $U$ of $x$, there exists $f \in \mathcal{A}$ such that $f(x) \equiv 1$ on a neighborhood of $x$ and $\operatorname{supp} f \subset U$.

Property 2: If $f, g \in \mathcal{A}$ satisfy $f(x) \leq g(x)$ for every $x \in X$, and $\operatorname{supp} f \subset$ $\operatorname{coz} g$, then there exists $k \in \mathcal{A}$ such that $f=g k$.

Property 3: For each $x \in X$, the set $(0,1) \cap\{f(x): f \in \mathcal{A}\}$ is nonempty.

It is easy to see that the image by a standard multiplicative bijection of a semigroup satisfying Properties 1, 2, and 3 is a semigroup that also satisfies them. One such semigroup is, for instance, that of all continuously differentiable functions on $I$ (also taking values in $I$ ). The general form of multiplicative bijections between this kind of semigroup will be given in Theorem 2.1. Finally we also give an example where Marovt's result does not hold for multiplicative bijections defined between general semigroups of this type, even when the ground spaces are first countable (see Example 3.5).

Suppose that $\varphi: \mathcal{A} \longrightarrow \mathcal{B}$ is multiplicative and bijective, where $\mathcal{A} \subset C(X, I)$ and $\mathcal{B} \subset C(Y, I)$ are semigroups satisfying Properties 1, 2, and 3. We say that $y \in Y$ is a standard point for $\varphi$ if there exist a number $p \in(0,+\infty)$ and a point $x \in X$ such that $\varphi(f)(y)=f(x)^{p}$ for every $f \in \mathcal{A}$. We denote by $\mathcal{R}(\varphi)$ the set of standard points for $\varphi$.

Throughout we assume that $X$ and $Y$ are compact and Hausdorff spaces. Given a (completely regular) space $Z$, we denote by $\beta Z$ its Stone-Čech compactification.

Finally we remark that our results are essentially different from those given in [5] for multiplicative bijections between spaces $C(X, \mathbb{R})$ of real-valued continuous functions on $X$. Even so, there is a similarity in that the multiplicative structure of the spaces of functions determines the space $X$ up to homeomorphism. More recent results in this line, concerning multiplicative maps on $C(X, \mathbb{R})$, are given for instance in [2] and [3].

\section{MAIN RESULTS}

We first give a theorem that provides a description of multiplicative bijections between semigroups satisfying the above properties. Recall that a subset $Z$ of $Y$ is a cozero-set if there exists a real-valued continuous function $f$ on $Y$ such that $Z=\{y \in Y: f(y) \neq 0\}$.

Theorem 2.1. Suppose that $\varphi: \mathcal{A} \longrightarrow \mathcal{B}$ is multiplicative and bijective, where $\mathcal{A} \subset C(X, I)$ and $\mathcal{B} \subset C(Y, I)$ are semigroups satisfying Properties 1, 2, and 3. Then

(1) there exist a homeomorphism $\mu$ from $Y$ onto $X$ and a continuous map $p: \mathcal{R}(\varphi) \longrightarrow(0,+\infty)$ such that

$$
\varphi(f)(y)=f(\mu(y))^{p(y)}
$$

for every $f \in \mathcal{A}$ and $y \in \mathcal{R}(\varphi)$;

(2) the set $\mathcal{R}(\varphi)$ is a dense cozero-set in $Y$, and the map $p$ can be extended to a continuous map from $Y$ into $[0,+\infty]$ taking values in $\{0,+\infty\}$ at every point of $Y \backslash \mathcal{R}(\varphi)$. 
Remark 2.1. Property 3 is necessary to ensure that Theorem 2.1 holds. For instance if we take $X=Y=[-1,1]$ and $\mathcal{A}:=\{f \in C(X, I): f(0) \in\{0,1\}\}$, it is clear that $\mathcal{A}$ satisfies both Properties 1 and 2 and that the map $\varphi: \mathcal{A} \longrightarrow \mathcal{A}$ defined for every $f \in \mathcal{A}$ as $\varphi(f)(x):=f(x)$ if $x \leq 0$ and $\varphi(f)(x):=f(x)^{2}$ if $x>0$ is a multiplicative bijection. Nevertheless, $\mathcal{R}(\varphi)=X$, so each point is standard for $\varphi$, but we cannot find a continuous map $p$ as given in Theorem 2.1

We next state the theorem characterizing all spaces on which can be defined a multiplicative bijection that is not standard. Recall that a topological space $Z$ is said to be pseudocompact if every real-valued continuous map on $Z$ is bounded.

Theorem 2.2. There exists a bijective and multiplicative map $\varphi: C(X, I) \longrightarrow$ $C(Y, I)$ that is not standard if and only if $X$ and $Y$ are homeomorphic and there exists a subset $Z$ of $Y$, not pseudocompact, such that $Y=\beta Z$.

Obviously Theorem 2.2 gives an answer in the negative to Marovt's conjecture. It is now enough to take any completely regular space $Z$ (thus ensuring that its Stone-Čech compactification exists) that is not pseudocompact (as for instance $\mathbb{N}$, $\mathbb{R}$, or any unbounded subset of a normed space), and we have that there are always multiplicative bijections on $C(\beta Z, I)$ that are not standard.

\section{Some other Results AND PROOFs}

The following is a key lemma to prove Theorem 2.1.

Lemma 3.1. Suppose that $u: \mathscr{A}_{1} \longrightarrow \mathscr{A}_{2}$ is an order preserving multiplicative bijection, where $\mathscr{A}_{1}$ and $\mathscr{A}_{2}$ are semigroups contained in $(0,1)$. Then there exists $p \in(0,+\infty)$ such that $u(\gamma)=\gamma^{p}$ for every $\gamma \in \mathscr{A}_{1}$.

Proof. Suppose on the contrary that there exist $\alpha, \beta \in \mathscr{A}_{1}$ such that $u(\alpha)=\alpha^{p}$ and $u(\beta)=\beta^{q}$, where $0<p<q$. By taking integer powers of $\alpha$ if necessary, we may assume without loss of generality that $\alpha<\beta$. Define $a:=-\log \alpha$ and $b:=-\log \beta$. Obviously $0<b<a$, and we can find natural numbers $n, m$ such that

$$
\frac{b}{a}<\frac{n}{m}<\frac{q}{p} \frac{b}{a} .
$$

Now it is clear that $m b<n a$ and $n p a<m q b$. These inequalities lead easily to $\alpha^{n}<\beta^{m}$ and $\alpha^{n p}>\beta^{m q}$, that is, $u\left(\alpha^{n}\right)>u\left(\beta^{m}\right)$, against the fact that $u$ is order preserving.

Proof of Theorem 2.1. (11) For each $x \in X$, let $\mathscr{U}_{x}$ be the set of all $f \in \mathcal{A}$ for which there exists a neighborhood of $x$ where $f \equiv 1$.

Let us see that $\mathscr{C}_{x}:=\left\{y \in Y: \varphi(f)(y)=1 \forall f \in \mathscr{U}_{x}\right\}$ is nonempty. First notice that, given any $f \in \mathscr{U}_{x}$, the set $\varphi(f)^{-1}(\{1\})$ is compact. On the other hand, given $f_{1}, \ldots, f_{n} \in \mathscr{C}_{x}$, we can find $f_{0} \in \mathcal{A}, f_{0} \neq 0$, such that $\operatorname{supp} f_{0} \subset\{z \in X$ : $\left.\prod_{i=1}^{n} f_{i}(z)=1\right\}$. This obviously implies that $f_{0} f_{i}=f_{0}$ for each $i$, so $\varphi\left(f_{0}\right) \varphi\left(f_{i}\right)=$ $\varphi\left(f_{0}\right) \neq 0$. We deduce that $\bigcap_{i=1}^{n} \varphi\left(f_{i}\right)^{-1}(\{1\}) \neq \emptyset$. Then $\left\{\varphi(f)^{-1}(\{1\}): f \in \mathscr{U}_{x}\right\}$ satisfies the finite intersection property, and we conclude that $\mathscr{C}_{x}$ is nonempty.

On the other hand, $\varphi^{-1}$ is also multiplicative, so given any $y \in \mathscr{C}_{x}$, we have that the set $\mathscr{C}_{y}$ (defined in a similar way as $\mathscr{C}_{x}$ ) is nonempty. Let us see that $\mathscr{C}_{y}=\{x\}$. Suppose that there exists $z \in \mathscr{C}_{y}, z \neq x$. Then we can find $f_{z} \in \mathscr{U}_{x}$ such that $z \notin \operatorname{supp} f_{z}$. Since $z \in \mathscr{C}_{y}$ and $\varphi\left(f_{z}\right)(y)=1$, then we can find $k \in \mathcal{B}$ 
with $\operatorname{supp} k \subset \operatorname{coz} \varphi\left(f_{z}\right)$ and $\varphi^{-1}(k)(z) \neq 0$. Clearly, if we now take $g \in \mathscr{U}_{z}$ with $f_{z} g=0$, then $g \varphi^{-1}(k) \neq 0$, but $\varphi(g) k=0$, which is impossible.

The above process lets us define a map $\mu: Y \longrightarrow X$, which turns out to be bijective, such that $\mu(y)$ is the only point in $\mathscr{C}_{y}$, and $y$ is the only point in $\mathscr{C}_{\mu(y)}$, for each $y \in Y$.

We next prove that $\mu$ is continuous at every point of $Y$ (and is consequently a homeomorphism). Take any $y \in Y$, and let $U$ be an open neighborhood of $\mu(y)$. We will see that if $f \in \mathscr{U}_{\mu(y)}$ and $\operatorname{supp} f \subset U$, then $\mu(\operatorname{coz} \varphi(f))$ is contained in $U$. Otherwise there exists $z \in Y$ such that $\varphi(f)(z) \neq 0$ and $\mu(z) \notin \operatorname{supp} f$, so we can take $k \in \mathscr{U}_{\mu(z)}$ such that $k f=0$. Obviously $\varphi(k)(z) \varphi(f)(z) \neq 0$, which is absurd.

Finally, we have that, by definition, if $y \in \mathcal{R}(\varphi)$, then there exist $p(y) \in(0,+\infty)$ and $x \in X$ such that $\varphi(f)(y)=f(x)^{p(y)}$ for every $f \in \mathcal{A}$. It is easy to check that $x=\mu(y)$. As for the map $p: \mathcal{R}(\varphi) \longrightarrow(0,+\infty)$, we have that for each $y \in \mathcal{R}(\varphi)$,

$$
p(y)=\frac{\log \varphi(f)(y)}{\log f(\mu(y))}
$$

for every $f \in \mathcal{A}$ with $f(\mu(y)) \neq 0,1$. This implies that $p$ is continuous at $y$ and consequently on $\mathcal{R}(\varphi)$.

(2) For each $y \in Y$, let $\mathcal{B}_{y}:=\{g(y): g \in \mathcal{B}\} \cap(0,1)$, and define $\mathcal{A}_{x}$ for each $x \in X$ in a similar way. Consider also the set $\mathcal{R}_{1}(\varphi)$ of all $y \in Y$ such that $\varphi(f)(y) \neq 0,1$ whenever $f \in \mathcal{A}$ satisfies $f(\mu(y)) \neq 0,1$. We need the following claim.

Claim. Let $y \in \mathcal{R}_{1}(\varphi)$. If $f, g \in \mathcal{A}$ satisfy $g(\mu(y)) \leq f(\mu(y))$, then $\varphi(g)(y) \leq$ $\varphi(f)(y)$. Moreover $\mu(y)$ belongs to $\mathcal{R}_{1}\left(\varphi^{-1}\right)$.

Suppose first that $g(\mu(y))<f(\mu(y))$, and take a neighborhood $U$ of $\mu(y)$ with $g(x)<f(x)$ for every $x \in U$. We pick $f_{0}, g_{0} \in \mathscr{U}_{\mu(y)}$ such that supp $f_{0} \subset U$ and such that supp $g_{0} \subset\left\{x \in X: f_{0}(x)=1\right\}$, respectively. Since $\mathcal{A}$ satisfies Property 2, then there exists $k \in \mathcal{A}$ such that $\left(f f_{0}\right) k=g g_{0}$. Also $k(\mu(y)) \in(0,1)$, and consequently

$$
\varphi(g)(y)=\varphi\left(g g_{0}\right)(y)=\varphi\left(f f_{0}\right)(y) \varphi(k)(y)<\varphi\left(f f_{0}\right)(y)=\varphi(f)(y) .
$$

We now prove that $\mu(y)$ belongs to $\mathcal{R}_{1}\left(\varphi^{-1}\right)$. Let $h \in \mathcal{B}$ be such that $h(y) \neq 0,1$. Suppose that $\varphi^{-1}(h)(\mu(y))=0$ and take any $l \in \mathcal{A}$ with $l(\mu(y)) \neq 0,1$, and $n \in \mathbb{N}$ such that $(\varphi(l)(y))^{n}<h(y)$. We then have that $\varphi^{-1}(h)(\mu(y))<l^{n}(\mu(y))$ and $h(y)>\varphi\left(l^{n}\right)(y)$, which goes against what we have proved above. We deduce that $\varphi^{-1}(h)(\mu(y)) \neq 0$. In a similar way we can deduce that $\varphi^{-1}(h)(\mu(y)) \neq 1$. Thus, $\mu(y)$ belongs to $\mathcal{R}_{1}(\varphi)$.

Now, working with $\varphi^{-1}$, we see clearly that if $g(\mu(y)) \leq f(\mu(y))$, then we cannot get $\varphi(g)(y)>\varphi(f)(y)$. The claim is proved.

For any $y \in \mathcal{R}_{1}(\varphi)$, we may define a map $\varphi_{y}: \mathcal{A}_{\mu(y)} \longrightarrow \mathcal{B}_{y}$ in the following way. Given $\alpha \in \mathcal{A}_{\mu(y)}$, there exists $f \in \mathcal{A}$ such that $f(\mu(y))=\alpha$. Then define $\varphi_{y}(\alpha):=\varphi(f)(y)$. It is clear by the above claim that $\varphi_{y}$ is well defined, and obviously it is multiplicative, order preserving, and bijective. Also, we have that $\varphi(f)(y)=1$ whenever $f(\mu(y))=1$ and $\varphi(f)(y)=0$ whenever $f(\mu(y))=0, f \in \mathcal{A}$. Consequently, by Lemma 3.1 we have that $\mathcal{R}_{1}(\varphi) \subset \mathcal{R}(\varphi)$. The other inclusion is immediate, so $\mathcal{R}_{1}(\varphi)=\mathcal{R}(\varphi)$.

We prove that $\mathcal{R}(\varphi)$ is dense in $Y$. Let $W_{0} \subset Y$ be open (and nonempty). Pick $y \in W_{0}$ and assume that $y \notin \mathcal{R}(\varphi)=\mathcal{R}_{1}(\varphi)$, so there exists $f_{0} \in \mathcal{A}$ such that $f_{0}(\mu(y)) \neq 0,1$ and $\varphi\left(f_{0}\right)(y) \in\{0,1\}$. Let $V:=\left\{x \in X: 0<f_{0}(x)<1\right\}$. We next see that $y$ does not belong to the interior of the set $W_{1}:=\left\{z \in W_{0}: \varphi\left(f_{0}\right)(z)=0\right\}$. 
Otherwise, we can find $g_{0} \in \mathscr{U}_{y}$ with $g_{0} \varphi\left(f_{0}\right)=0$. This implies that $\varphi^{-1}\left(g_{0}\right) f_{0}=0$, against the fact that $\varphi^{-1}\left(g_{0}\right)(\mu(y))=1$ and $f_{0}(\mu(y)) \neq 0$. On the other hand, $y$ does not belong to the interior of the set $W_{2}:=\left\{z \in W_{0}: \varphi\left(f_{0}\right)(z)=1\right\}$, because $\varphi\left(f_{0}\right) \notin \mathscr{U}_{y}$. Consequently, due to the form of $W_{1}$ and $W_{2}$, we have that $y$ belongs to the closure of $Y \backslash\left(W_{1} \cup W_{2}\right)$. This means that

$$
W:=\mu^{-1}(V) \cap\left(W_{0} \backslash\left(W_{1} \cup W_{2}\right)\right) \neq \emptyset .
$$

As in the proof of the claim, it is clear that given any point in $Y$, and $f, g \in \mathcal{A}$ with $g(\mu(y))<f(\mu(y))$, then $\varphi(g)(y) \leq \varphi(f)(y)$. Now it is straightforward to check that every point in $W$ belongs to $\mathcal{R}_{1}(\varphi)=\mathcal{R}(\varphi)$.

We finally see that $\mathcal{R}(\varphi)$ is a cozero-set. Suppose that $y \in Y \backslash \mathcal{R}(\varphi)$. Take any net $\left(z_{\alpha}\right)_{\alpha \in \Lambda}$ in $\mathcal{R}(\varphi)$ converging to $y$. Clearly, since $y \notin \mathcal{R}_{1}(\varphi)$, there exists $f \in \mathcal{A}$ with $f(\mu(y)) \neq 0,1$ and either $\varphi(f)(y)=0$ or 1 . Also

$$
p\left(z_{\alpha}\right)=\frac{\log \varphi(f)\left(z_{\alpha}\right)}{\log f\left(\mu\left(z_{\alpha}\right)\right)}
$$

for every $\alpha \in \Lambda$. The conclusion that $\lim _{\alpha} p\left(z_{\alpha}\right) \in\{0,+\infty\}$ follows easily. Finally, it is clear that $\mathcal{R}(\varphi)$ coincides with the cozero-set of the continuous function $Y \longrightarrow \mathbb{R}$ given by

$$
y \mapsto p(y)\left(p(y)^{2}+1\right)^{-1}
$$

if $y \in \mathcal{R}(\varphi)$, and $y \mapsto 0$ otherwise.

Remark 3.1. If in Theorem 2.1 we assume that the semigroups satisfy only Properties 1 and 2, a similar proof ensures the existence of the homeomorphism $\mu$ between $Y$ and $X$, even if the theorem does not hold (see Remark 2.1).

In what follows, when we want to specify the homeomorphism $\mu$ and the map $p$ corresponding to a multiplicative and bijective map $\varphi$, as given in Theorem 2.1. we will write $\varphi[\mu, p]$. It is obvious that if $\varphi=\varphi[\mu, p]$, then $\varphi^{-1}=\left[\mu^{-1}, q\right]$, where $q=1 /\left(p \circ \mu^{-1}\right)$.

Corollary 3.2. Let $\varphi: \mathcal{A} \longrightarrow \mathcal{B}$ be multiplicative and bijective, where $\mathcal{A} \subset C(X, I)$ and $\mathcal{B} \subset C(Y, I)$ are semigroups satisfying Properties 1, 2, and 3. If $\mathcal{R}(\varphi)$ is pseudocompact, then $\mathcal{R}(\varphi)=Y$.

Proof. It is obvious that if $\varphi=\varphi[\mu, p]$ and $\varphi^{-1}=\varphi^{-1}\left[\mu^{-1}, q\right]$, then both $p$ and $q$ are bounded, and consequently neither $+\infty$ nor 0 is a limit point of $p(\mathcal{R}(\varphi))$. The conclusion follows from Theorem 2.1.

Proposition 3.3. Let $\varphi: C(X, I) \longrightarrow C(Y, I)$ be multiplicative and bijective. Then $Y=\beta(\mathcal{R}(\varphi))$.

Proof. We follow the notation given after Remark 3.1. Suppose that $\varphi=\varphi[\mu, p]$. It is clear that if $\mathbf{1}$ is the map constantly equal to 1 on $X$ and we consider the multiplicative bijection $\psi=\psi\left[\mu^{-1}, \mathbf{1}\right]: C(Y, I) \longrightarrow C(X, I)$, then the composition $\varphi \circ \psi: C(Y, I) \longrightarrow C(Y, I)$ is $\varphi \circ \psi\left[\mathbf{i d}_{Y}, p\right]$ (where $\mathbf{i d}_{Y}$ is the identity map on $Y$ ). Also, $\mathcal{R}_{1}(\varphi)=\mathcal{R}_{1}(\varphi \circ \psi)$, so $\mathcal{R}(\varphi)=\mathcal{R}(\varphi \circ \psi)$ (see the proof of Theorem 2.1). Consequently we can assume without loss of generality that $X=Y$ and $\mu=\mathbf{i d}_{Y}$.

Notice that if we consider the continuous extensions of $p$ and $q$ to maps from $Y$ to $[0,+\infty]$, as seen in Theorem 2.1 then $p^{-1}(\{0\})=q^{-1}(\{+\infty\})$ and $p^{-1}(\{+\infty\})=$ $q^{-1}(\{0\})$. Thus, assuming that $\beta(\mathcal{R}(\varphi)) \neq Y$, at least one of the sets $p^{-1}(\{0\})$, 
$q^{-1}(\{0\})$ is nonempty. We conclude that there is a continuous map from $Y \backslash$ $p^{-1}(\{0\})$, or from $Y \backslash q^{-1}(\{0\})$, to $I$ which does not admit a continuous extension to $Y$.

Taking into account that $\mathcal{R}(\varphi)=\mathcal{R}\left(\varphi^{-1}\right)$, we assume without loss of generality that there exists a continuous function $g_{0}: Y \backslash p^{-1}(\{0\}) \longrightarrow I$ that cannot be continuously extended to $Y$. We consider the map $h \in C(Y, I)$ whose image by $\varphi$ is the constant function $1 / e$. It is easily seen that since $p(y) \log h(y)=\log \varphi(h)(y)$ for every $y \in \mathcal{R}(\varphi)$,

$$
h(y)=\exp \left(-\frac{1}{p(y)}\right)
$$

if $y \in Y \backslash p^{-1}(\{0\})$ and $h \equiv 0$ on $p^{-1}(\{0\})$.

Since $p^{-1}(\{0\})$ is a zero-set, there exists a sequence $\left(K_{n}\right)$ of compact subsets of $Y$ with $K_{n+1}$ contained in the interior of $K_{n}$ for each $n \in \mathbb{N}$ and such that $p^{-1}(\{0\})=\bigcap_{n=1}^{\infty} K_{n}$. For each $n \in \mathbb{N}$, we take $g_{n} \in C(Y, I)$ satisfying $g_{n} \equiv g_{0}$ on $Y \backslash K_{n}$. We denote by $f_{n}$ its counterimage by $\varphi$.

Notice that if we define $f_{0}(z)=f_{n}(z)$ whenever $z \notin K_{n}$, then $f_{0}: Y \backslash p^{-1}(\{0\}) \longrightarrow$ $I$ is continuous. It is also obvious that $f_{0} h$ (defined as 0 on $\left.p^{-1}(\{0\})\right)$ belongs to $C(Y, I)$. On the other hand, we have that $f_{0} h \equiv f_{n} h$ outside $K_{n}$ for each $n \in \mathbb{N}$ and consequently

$$
\varphi\left(f_{0} h\right) \equiv \varphi\left(f_{n} h\right) \equiv g_{n} \varphi(h) \equiv g_{0} \varphi(h)
$$

outside each $K_{n}$. This implies that $\varphi\left(f_{0} h\right) \equiv g_{0} \varphi(h)$ on $Y \backslash p^{-1}(\{0\})$. Now it is easy to see that since $\varphi(h) \equiv 1 / e$ on $p^{-1}(\{0\}), \varphi\left(f_{0} h\right)$ is not defined at some points of $p^{-1}(\{0\})$, which is absurd.

Remark 3.2. We deduce in particular that if the set $Y \backslash \mathcal{R}(\varphi)$ is nonempty, then its cardinality is at least $2^{\mathfrak{c}}$. Also, if it is endowed with the restricted topology, then no point in $Y \backslash \mathcal{R}(\varphi)$ is a $G_{\delta}$ (see [1, Chapter 9]).

Remark 3.3. By Remark 3.2, we have that each point of $Y$ having a countable base of neighborhoods belongs to $\mathcal{R}(\varphi)$ for every $\varphi$. In particular, if $Y$ is first countable, then $\mathcal{R}(\varphi)=Y$, which is essentially Marovt's result. But there can be spaces which are not first countable and for which every point is standard. As an easy example, consider a bijective and multiplicative map $\varphi: C\left(\left[0, \omega_{1}\right], I\right) \longrightarrow$ $C\left(\left[0, \omega_{1}\right], I\right)$, where $\omega_{1}$ denotes the first noncountable ordinal. Since each point of $\left[0, \omega_{1}\right)$ has a countable base of neighborhoods, we deduce that $\left[0, \omega_{1}\right] \backslash \mathcal{R}(\varphi) \subset\left\{\omega_{1}\right\}$, and again by Remark 3.2. we cannot have $\left[0, \omega_{1}\right] \backslash \mathcal{R}(\varphi)=\left\{\omega_{1}\right\}$. We conclude that $\mathcal{R}(\varphi)=\left[0, \omega_{1}\right]$.

Remark 3.4. The conclusion given in Remark 3.3 is not true for more general semigroups; that is, not every point having a countable base of neighborhoods necessarily belongs to $\mathcal{R}(\varphi)$ (see Example 3.5).

Using Theorem 2.1. we easily see that every isolated point is standard for every multiplicative bijection. More generally, the next result allows us to identify some points that belong to $\mathcal{R}(\varphi)$ for every $\varphi$.

Corollary 3.4. Let $y \in Y$ be such that $\beta(Y \backslash\{y\}) \neq Y$. Then $y \in \mathcal{R}(\varphi)$ for every multiplicative bijection $\varphi: C(X, I) \longrightarrow C(Y, I)$.

Proof. Suppose that $y \notin \mathcal{R}(\varphi)$. Thus, taking into account Proposition 3.3 and the fact that $\mathcal{R}(\varphi) \subset Y \backslash\{y\}$, we deduce that $\beta(Y \backslash\{y\})=Y$, and we are finished. 
Remark 3.5. Obviously, the converse of Corollary 3.4 is in general not true. For an easy example, consider for instance the point $\omega_{1}$ in Remark 3.3. and take into account that $\beta\left[0, \omega_{1}\right)=\left[0, \omega_{1}\right]$.

Remark 3.6. Marovt's result cannot be given in general for the kind of semigroups we are dealing with, even if the ground spaces are assumed to be first countable. That is, it is possible that $\mathcal{R}(\varphi) \neq X$ for a multiplicative bijection $\varphi: \mathcal{A} \longrightarrow \mathcal{B}$, even in the case when $\mathcal{A}, \mathcal{B} \subset C(X, I)$ satisfy Properties 1,2 , and 3 , and the space $X$ is first countable. We next include an example of this fact.

Example 3.5. For a topological space $Z$, denote by $C\left(Z, \mathbb{R}_{\geq 0}\right)$ the set of all continuous maps from $Z$ to $[0,+\infty)$. Let $\mathbb{N} \cup\{\infty\}$ be the one-point compactification of $\mathbb{N}$ and let $\varphi: C\left(\mathbb{N} \cup\{\infty\}, \mathbb{R}_{\geq 0}\right) \longrightarrow C\left(\mathbb{N}, \mathbb{R}_{\geq 0}\right)$ be defined, for each $f \in C\left(\mathbb{N} \cup\{\infty\}, \mathbb{R}_{\geq 0}\right)$, as $\varphi(f)(n):=f(n)^{n}$ for every $n \in \mathbb{N}$. It is clear that $\varphi$ is multiplicative and injective. Since it also preserves order, then the image of each $f \in C(\mathbb{N} \cup\{\infty\}, I)$ takes values in $I$.

Consider now the subset $\mathcal{A}_{1}$ of $C(\mathbb{N} \cup\{\infty\}, I)$ of all characteristic functions which are continuous, and let $f_{0} \in C(\mathbb{N} \cup\{\infty\}, I)$ be the counterimage by $\varphi$ of the constant function equal to $1 / 2$. Define $\mathcal{A}$ as the set of all functions of the form $f=\alpha g f_{0}^{m}$ for some $\alpha \geq 0, g \in \mathcal{A}_{1}$, and $m \in \mathbb{Z}$, such that $f(n) \in I$ for every $n \in \mathbb{N}$.

It is easy to see that if $f \in \mathcal{A}$, then $\lim _{n \longrightarrow \infty} \varphi(f)(n)$ exists, so in a natural way we may define a map $\varphi: \mathcal{A} \longrightarrow C(\mathbb{N} \cup\{\infty\}, I)$. Put $\mathcal{B}:=\varphi(\mathcal{A})$. It is easy to see that $\mathcal{A}$ and $\mathcal{B}$ satisfy Properties 1,2 , and 3 . On the other hand, every point in $\mathbb{N}$ is isolated, and consequently $\mathbb{N}$ is contained in $\mathcal{R}(\varphi)$. Nevertheless, $\infty$ does not belong to $\mathcal{R}(\varphi)$.

Proof of Theorem 2.2. Suppose that there exists a multiplicative bijection $\varphi: C(X, I) \longrightarrow C(Y, I)$ that is not standard. First, by Theorem 2.1, $X$ and $Y$ must be homeomorphic. Also, calling $Z:=\mathcal{R}(\varphi)$, we have by Corollary 3.2 that $Z$ is not pseudocompact, and by Proposition 3.3 that $Y=\beta Z$.

Conversely, suppose that $X$ and $Y$ are homeomorphic and that there exists a proper subset $Z$ of $Y$ which is not pseudocompact and such that $Y=\beta Z$. It is clear that without loss of generality we may assume that $X=Y$. Since $Z$ is not pseudocompact, there exists an unbounded continuous function $u: Z \longrightarrow[1,+\infty)$. We define a map $\varphi: C(Z, I) \longrightarrow C(Z, I)$ as $\varphi(g)(z):=g(z)^{u(z)}$ for each $g \in C(Z, I)$ and $z \in Z$.

It is easy to check that $\varphi$ is multiplicative and injective. Let us see that $\varphi$ is surjective. To this end, we take any $k \in C(Z, I)$, and consider the map $L_{k}$ : $Z \longrightarrow[-\infty, 0]$ defined as $L_{k}(z):=(\log k(z)) / u(z)$ if $k(z) \neq 0$, and $L_{k}(z):=-\infty$ if $k(z)=0$. It is easy to check that $L_{k}$ is continuous and that $\varphi\left(\exp L_{k}\right)=k$ (assuming $\exp (-\infty)=0$ ).

Obviously $\varphi$ can be seen as a multiplicative bijection on $C(\beta Z, I)$. On the other hand, if for some homeomorphism $\mu: \beta Z \longrightarrow \beta Z$ and a continuous map $v: \beta Z \longrightarrow(0,+\infty), \varphi(f)(x)=f(\mu(x))^{v(x)}$ for every $f$, then $\mu$ must be the identity on $\beta Z$, and $v(z)=u(z)$ for every $z \in Z$. This obviously implies that $v$ must attain the value $+\infty$ on some points of $\beta Z \backslash Z$, which is absurd.

\section{ACKNOWLEDGEMENTS}

The author wishes to thank the referee for valuable remarks and, also, Ana M. Ródenas for drawing his attention to this subject. 


\section{REFERENCES}

[1] L. Gillman and M. Jerison, Rings of continuous functions. Van Nostrand, Princeton, NJ, 1960. MR0116199 (22:6994)

[2] H. Goldmann and P. Semrl, Multiplicative derivations on $C(X)$. Monatsh. Math. 121 (1996), 189-197. MR $1383530(96 \mathrm{~m}: 46041)$

[3] G. Lešnjak and P. Semrl, Continuous multiplicative mappings on $C(X)$. Proc. Amer. Math. Soc. 126 (1998), 127-133. MR.1402871 (98c:46102)

[4] J. Marovt, Multiplicative bijections of $\mathcal{C}(\mathcal{X}, I)$. Proc. Amer. Math. Soc. 134 (2006), 1065-1075. MR2196040 (2006k:46078)

[5] A. N. Milgram, Multiplicative semigroups of continuous functions. Duke Math. J. 16 (1940), 377-383. MR0029476 (10:612a)

[6] L. Molnár, Sequential isomorphisms between the sets of von Neumann algebra effects. Acta Sci. Math. (Szeged) 69 (2003), 755-772. MR2034206 (2005d:46128)

Departamento de Matemáticas, Estadística y Computación, Universidad de Cantabria, Facultad de Ciencias, Avda. de los Castros, s. N., E-39071 Santander, Spain

E-mail address: araujoj@unican.es 